\section{NSCLC: ERCC1 und RRM1 als molekulare Marker für das Therapieansprechen}

\begin{abstract}
Auf die Tumorbiologie abgestimmte Therapien können das Gesamtüberleben von Krebspatienten deutlich verbessern. Dies gilt auch für Patienten mit nichtkleinzelligem Lungenkarzinom (NSCLC). Dabei werden zunehmend Biomarker identifiziert, die eine adaptierte Chemotherapie ermöglichen.
\end{abstract}

$B^{\mathrm{i}}$ iomarker-Analysen zweier randomisierter Phase-III-Studien ergaben, dass Patienten, die in situ erhöhte Levels des DNA-Reparaturproteins ERCC1 aufweisen, von einer platinbasierten Chemotherapie profitieren, und solche mit hohen Spiegeln an Ribonukleotidreduktase M1 (RRM1) gut auf Gemcitabin ansprechen. In einer internationalen Phase-III-Studie mit 275 NSCLC-Patienten in fortgeschrittenen Stadien untersuchten Gerold Bepler und Kollegen nun inwieweit Patienten von Therapieschemata profitieren, die sich an den ERCC1- und RRM1-Expressionsprofilen orientierten: Im experimentellen Arm richtete sich die Therapie nach den RRM1- und ERCC1-Levels:

-RRM1 $\downarrow$ und ERCC1 $\downarrow$ : Gemcitabin/ Carboplatin

\section{_RRM1 $\uparrow$ und ERCC1 $\downarrow$ : Docetaxel/ Carboplatin \\ —RRM1 $\downarrow$ und ERCC1 $\uparrow:$ Gemcitabin/ Docetaxel \\ _RRM1 $\uparrow$ und ERCC1 $\uparrow:$ Docetaxel/ \\ Vinorelbin}

Der Kontrollarm erhielt Gemcitabin/ Carboplatin unabhängig vom ERCC1und RRM1-Expressionsmuster.

Insgesamt ergaben sich keine signifikanten Unterschiede in beiden Studienarmen bzgl. des progressionsfreien Überlebens (6,1 vs. 6,9 Monate) und Gesamtüberlebens (11,0 vs. 11,3 Monate). Verglich man Patienten mit niedrigen Levels an beiden Proteinen und identischer Therapie profitierte der Kontrollarm mit einem signifikant günstigeren PFS gegenüber dem experimentellen Arm (8,1 vs. 5,0 Monate).

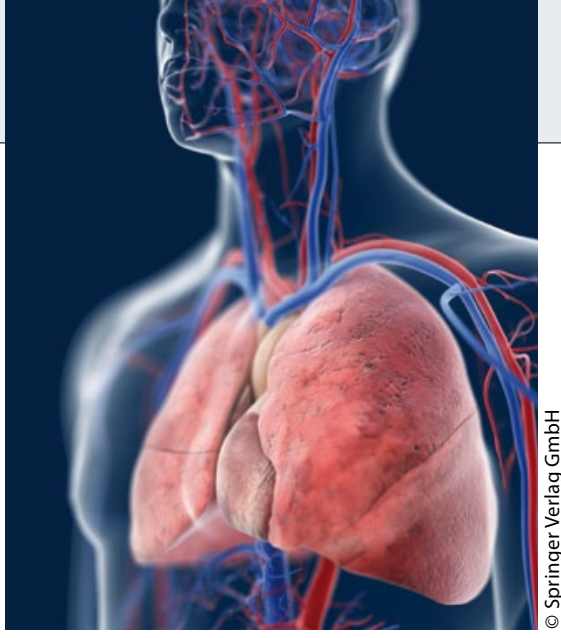

Therapieoptimierung bei Tumoren der Lunge mithilfe von Biomarkern.

Fazit: Protein-Expressionsanalysen als Grundlage für die Therapieentscheidung bei Patienten mit neu diagnostiziertem NSCLC in fortgeschrittenem Stadium erwiesen sich in dieser Studie als praktikabel. Es müssten scheinbar aber noch die Spezifität der Analyse-Assays verbessert und die Aufbereitungen der Proben optimiert und standardisiert werden, bevor weitere Studien dieser Art durchgeführt werden, so die Autoren. Ihrer Meinung nach gab es bei den Analysen falsch-negative Ergebnisse. Wolfgang Zimmermann

Bepler G et al. Randomized International Phase III Trial of ERCC1 and RRM1 Expression-Based Chemotherapy Versus Gemcitabine/Carboplatin in Advanced Non-Small-Cell Lung Cancer. J Clin Oncol. 2013;31(19):2404-12.

\title{
Tyrosinkinaseinhibitoren beim NSCLC mit EGFR-Mutation
}

\begin{abstract}
Mutationen der Rezeptorproteinkinase EGFR (epidermal growth factor receptor) spielen eine Schlüsselrolle in der Tumorgenese und Progression des nichtkleinzelligen Lungenkarzinoms. Einer aktuellen Metaanalyse zufolge sollte vor der Behandlung immer auch der EGFR-Mutationsstatus bestimmt werden.
\end{abstract}

$\mathrm{D}$ ie Einführung zielgerichteter Agenzien markierte einen großen Fortschritt in Richtung „personalisierte Therapie“. Anlass für Kontroversen bietet die Umsetzung dieser Innovationen in den klinischen Alltag. Dies gilt auch für die Therapie mit Tyrosinkinaseinhibitoren (TKIs). Welchen Nutzen hat die Behandlung mit EGFR-Tyrosinkinaseinhibitoren (EGFR-TKI) auf das gesamte und progressionsfreie Überleben von Patienten mit einem fortgeschrittenen NSCLC mit und ohne EGFR-Mutationen. Dieser Frage gingen Chee Khoon Lee und Kollegen in einer Metaanalyse von 23 randomisierten Studien nach, in denen Mono- therapien mit EGFR-TKI oder die Kombination von EGFR-TKI mit Chemotherapien bzw. Placebo verglichen wurden. EGFR-TKI sind hoch aktiv bei Patienten deren Tumoren aktivierende Mutationen im EGFR-Gen aufweisen. Sie verglichen daher für ihre Analyse die Behandlungsergebnisse für die Subgruppen mit positivem $\left(\mathrm{EGFR}_{\text {mut }}\right)$ oder negativem EGFRMutationsstatus (EGFR mut- $_{\text {mat }}$ ).

EGFR-TKI behandelte NSCLC-Patienten mit nachgewiesenem $\mathrm{EGFR}_{\text {mut+ }}$ leben länger progressionsfrei als Patienten der Vergleichsgruppen. Der EGFRMutationsstatus erwies sich zudem als prädiktiv für das progressionsfreie
Überleben (PFS) in allen Settings. Dies verdeutlichen die Hazard Ratios für die Frontline-Therapie: 1,06 für EGFR mut- $_{\text {(= }}$ kürzere Progressionsfreiheit) und 0,43 für $\mathrm{EGFR}_{\text {mut+ }}$ (= längere Progressionsfreiheit). Wehrmutstropfen dabei: Die Behandlung mit TK verbesserte nicht das Gesamtüberleben der Patienten, unabhängig vom EGFR-Mutationsstatus.

Fazit: Die Autoren folgern: Bei Patienten mit NSCLC sollte immer der EGFR-Mutationsstatus bestimmt werden und EGFR-TKI sind als Frontline-Therapie beim fortgeschritten, EGFR ${ }_{\text {mut+ }}$ NSCLC, $\mathrm{zu}$ favorisieren, auch wenn das Gesamtüberleben nicht beeinflusst wird.

Wolfgang Zimmermann

Lee CK et al. Impact of EGFR inhibitor in non-small lung cancer on progression-free and overall survival: a meta-analysis. J Natl Cancer. 2013;105(9):595-605. 